\title{
Apoptotic Index Fails to Predict Chemoresponse in Breast Cancer
}

\section{Kamal Basri Siregar}

Division of Surgical Oncology, Department of Surgery, Faculty of Medicine, Universitas Sumatera Utara, Medan, North Sumatera, Indonesia.

\begin{abstract}
The responsiveness of neoadjuvant chemotherapy in Triple Negative Breast Cancer (TNBC) needs determination to prevent overtreatment with chemoresistance regimen. A total of 60 consented patients from Haji Adam Malik and Bunda Thamrin Hospital were included in this cohort prospectie study. Patients with heart, kidney, liver disease, history of surgery, chemotherapy, or hormonal therapy were excluded. Apoptotic indexes were taken from all subjects before and after neoadjuvant chemotherapy. After 3 cycles of neoadjuvant chemotherapy, 31 subjects $(51.7 \%)$ did not show clinical response while 29 subjects $(48.3 \%)$ had clinical response. There was no significant difference of apoptotic index before and after neoadjuvant chemotherapy $(5.47+1.38 \mathrm{vs} 5.52+1.08 \mathrm{pg} / \mathrm{mL})$. There was also no significant relationship between apoptosis index and clinical response $(\mathrm{p}=0.993)$. This study showed that apoptotic index fails to be neoadjuvant chemotherapy response predictor in triple negative breast cancer. Further research with larger samples is needed to confirm this result.
\end{abstract}

Keyword: Breast Cancer, Chemotherapy, Surgery

Received [19 February 2018] | Revised [30 May 2019] | Accepted [30 May 2019]

\footnotetext{
*Corresponding author at: Faculty of Medicine, Universitas Sumatera Utara, Medan, Indonesia

E-mail address: kamalbasrisiregar@yahoo.com DOI: https://doi.org/10.32734/sumej.v2i2.1062
} 


\section{Introduction}

Triple Negative Breast Cancer (TNBC) accounts for 17-21\% of breast cancer case [1]. but accounts for the worst prognosis of all breast cancers [2]. The absence of hormonal receptors makes chemotherapy an important role in the treatment of TNBC [3]. Multicentre studies showed that $36 \%$ of TNBC patients receiving neoadjuvant chemotherapy showed complete clinical response and a significant good prognosis for disease free survival $(\mathrm{p}=0.001)$ [4]. In the EORTC study, it was reported that almost $23 \%$ of patients being enabled for breast conserving surgery after neoadjuvant chemotherapy. However, there were also many chemoresistance cases making the neoadjuvant chemotherapy almost had no benefit but only yielded harmful side effects [5]. Biomarker for that can accurately identify a patient with sensitivity for chemotherapy is needed [6]. This study will examine the expression of apoptotic index (AI), which is commonly used as a measure of apoptotic or apoptotic cell count per 1000 tumor cells.

\section{Methodology}

This prospective cohort was conducted from June 2015 to February 2017. As many as 60 TNBC subjects undergoing surgery in Haji Adam Malik General Hospital and Bunda Thamrin Hospital were included in this study. They must approve the informed consent and had a Karnofsky scale $>70$. Exclusion criteria when there is morbidity of heart disease, kidney, liver, history of surgery, hormonal therapy, or previous chemotherapy. The parameters of this study were apoptotic index pre-chemotherapy and post- neoadjuvant chemotherapy. The TUNEL assayedapoptotic index were done to all subjects before and after neoadjuvant chemotherapy administration. The chemotherapy regimen were given based on the hospitals protocol in Medan, Indonesia. Data were then collected and analyzed by SPSS 17.0.

\section{Results}

A total of 60 TNBC patients followed the study until completion. Subjects ranged from 30 to 73 years old with the majority of premenopausal subjects $(56.7 \%)$. As many as $75 \%$ of patients diagnosed in stage IIIB and $43.3 \%$ had T3 tumor size, $80 \%$ IDC histology type, and $41.7 \%$ were in grade II. After 3 cycles of neoadjuvant chemotherapy, 31 subjects (51.7\%) did not show clinical response while 29 subjects (48.3\%) had clinical response (Table 1). 
Table 1 Demographics Data of Subjects

\begin{tabular}{ll}
\hline Characteristics & Percentage \\
\hline Age (years old) & $8(13.3 \%)$ \\
$<35$ & $11(18.3 \%)$ \\
$35-40$ & $16(26.7 \%)$ \\
$41-50$ & $19(31.7 \%)$ \\
$51-60$ & $6(10.0 \%)$ \\
$>60$ & \\
Menopausal status & $34(56.7 \%)$ \\
Premenopause & $26(43.3 \%)$ \\
Postmenopause & \\
Stage & $15(25.0 \%)$ \\
IIIA & $45(75.0 \%)$ \\
IIIB & \\
Tumor size & $3(5.0 \%)$ \\
Tx & $1(1.7 \%)$ \\
T1 & $26(43.3 \%)$ \\
T3 & $30(5.0 \%)$ \\
T4 & \\
Histological type & $48(80.0 \%)$ \\
IDC & $12(20.0 \%)$ \\
ILC & \\
Grade & $9(15 \%)$ \\
I & $26(43,33 \%)$ \\
II & $25(41,66 \%)$ \\
III & \\
Chemotherapy & \\
response & \\
Response & \\
No response & \\
\hline
\end{tabular}

In this study, the mean apoptotic index before neoadjuvant chemotherapy was $5.47+1.38 \mathrm{pg} / \mathrm{mL}$ and after neoadjuvant chemotherapy was $5.52+1.08 \mathrm{pg} / \mathrm{mL}$. The result of t-paired test showed that there was no significant difference between mean of AI postchemotherapy and preechemotherapy $(\mathrm{p}=0.819)$ with only increase of $0.05+1.68 \mathrm{pg} / \mathrm{mL}$ (Table 2). Most of the subjects in the group that responded nor responded clinically to chemotherapy showed an increased apoptotic index (51.7\%). However, Phi and Cramer's V correlation analysis showed no significant relationship between apoptosis index $(\mathrm{p}=0.993)$ and clinical response (Table 3).

Table 2 Mean Difference of Apoptotic Index Postchemotherapy and Prechemotherapy

\begin{tabular}{lllll}
\hline Parameter & $\begin{array}{l}\text { Pre-chemotherapy } \\
(\mathrm{pg} / \mathrm{mL})\end{array}$ & $\begin{array}{l}\text { Post-chemotherapy } \\
(\mathrm{pg} / \mathrm{mL})\end{array}$ & Difference & $p$ \\
\hline $\begin{array}{l}\text { Apoptotic } \\
\text { index }\end{array}$ & $5.47 \pm 1.38$ & $5.52 \pm 1.08$ & $0.05 \pm 1.68$ & 0.819 \\
\hline
\end{tabular}


Table 3 The Relationship Between Apoptotic Index and Neoadjuvant Chemotherapy Response

\begin{tabular}{lllll}
\hline $\begin{array}{l}\text { Apoptotic } \\
\text { index }\end{array}$ & \multicolumn{2}{l}{$\begin{array}{l}\text { Clinical Response of Neoadjuvant } \\
\text { Chemotherapy }\end{array}$} & \multirow{2}{*}{ Total } & p \\
\cline { 2 - 3 } & No Response & Good Response & & \\
\hline Unchange & $4(12.9 \%)$ & $4(6,7 \%)$ & $8(13.3 \%)$ & \\
Decrease & $11(18.3 \%)$ & $10(16,7 \%)$ & 21 & 0.993 \\
& & $15(25,0 \%)$ & $\begin{array}{l}31 \\
(35.0 \%)\end{array}$ & \\
Increase & $16(26.7 \%)$ & $29(48,3 \%)$ & $\begin{array}{l}60 \\
(51.7 \%)\end{array}$ & \\
& & & $(100 \%)$ & \\
\hline Total & $31(51.7 \%)$ & & & \\
& & & & \\
\end{tabular}

\section{Discussion}

The age-specific prevalence pattern for TNBC was not fully understood until SEER collected data from 1997-2002 [7]. In this study, subjects aged 27 to 73 years old that mostly aged between 51 and 60 years (31.7\%). Prevalence of subjects aged 35 to 50 years old was $76.7 \%$. This result was in accordance with SEER survey that found peak incidence cases of TNBC from 35-60 years, and afterwards not much different. The population based study by Ambrosone showed that 2.9 times the increased risk of TNBC in women at this age was due to unknown increase in the use of oral contraceptives [8]. However, there is a research controversy by Stark, who observed increased 1.9 times risk of TNBC occurred at younger ages. But, this can be due to the researchers comparing it with luminal breast cancer A [9]. Phipps in Americans showing that menopausal age was not associated with an increased risk of TNBC [10], as did other studies by Xing in China [11] and Yang in Poland [12] but increased the risk of luminal breast cancer A. Phipps even tried hormonal therapy on TNBC patients and found no improvement. The study also showed a nearly equal proportion between premenopausal and postmenopausal patients, $56.66 \%$ and $43.33 \%$, respectively [10].

Based on table 1, there were 31 samples (51.7\%) that did not respond to neoadjuvant chemotherapy and 29 samples $(48.3 \%)$ that response to neoadjuvant chemotherapy. This proportion was higher than that of Yarso (2012), which showed only 15\% of clinical responses [13]. Although Torrisi (2010) reported 77.5\% [14]. Von Minckwitz (2014) found that the addition of neoadjuvant chemo-therapy carboplatin to the regimen taxan, anthracyclin, and targeted therapies significantly increase the propor-tion of patients achieving a complete response. This suggested that neoadjuvant chemotherapy could reduce the size of the tumor and eradicated almost half the TNBC cases [15]. Otherwise, some unresponse patients to be overtreated because of the unpredictability of TNBC. This condition should be prevented by the discovery of prognostic factor that can predict neoadjuvant chemotherapy response earlier. 
Reviewing the apoptotic index, there was no significant mean difference between postoperative and postte-motherapy $(\mathrm{p}=0.819)$ and no significant differences were found between apoptotic index and TNBC chemo-therapy response ( $\mathrm{p}=0.993)$. The proportion of the increase or decrease in the apoptotic index was almost com-parable between those who responded and unrespond to neuroadjuvant TNBC. This result was similar to Yang (2001) which showed that maybe the apoptotic index low due to low concentration of doxorubicin given. In MCF-7 cells, 18-hour doxorubicin were exposed that resulted in caspase activation and other apoptotic sub-strates in line with the addition of 2-10 microM [16]. Unlike ODonovan (2003) study in 103 breast tissue samples, they showed that Caspase-3 precursor and active form were higher in breast cancer than normal tissue ( $\mathrm{p}=0.0188 ; \mathrm{p}=0.0002)$ [17]. Similarly, Sharma (2009) showed that tumor biology markers (Bcl-2, Apoptotic Index and Caspase-3) changes occurred 24-48 hours after first neoadjuvant chemotherapy cycles. These markers cpuld be as a factors to predict the response of chemotherapy, but to prove them statistically, need research with a larger sample size [18].

It has been shown in the previous data that there was no significant increase in apoptosis index after neoadju-vant chemotherapy. The author concluded that apoptotic index was not able to be a response predictor of chemo-therapy in TNBC. However, further research was suggested to investigate this issue using larger samples.

\section{Conclusion}

There was no significant difference between apoptotic index and clinical chemotherapy response to neoadjuvant chemotherapy on triple negative breast cancer. Further research with larger samples is needed to determine the role and pathway of chemotherapy induced caspase 3 rise.

\section{REFERENCES}

[1] J. Wu, S. Li, W. Jia, and F. Su, "Response and prognosis of taxanes and anthracyclines neoadjuvant chemotherapy in patients with triple-negative breast cancer," Journal of cancer research and clinical oncology, vol. 137, no. 10, p. 1505, 2011.

[2] W. D. Foulkes, I. E. Smith, and J. S. Reis-Filho, "Triple-negative breast cancer," New England journal of medicine, vol. 363, no. 20, pp. 1938-1948, 2010.

[3] C. Liedtke et al., "Response to neoadjuvant therapy and long-term survival in patients with triple-negative breast cancer," Journal of clinical oncology, vol. 26, no. 8, pp. 1275-1281, 2008.

[4] B. Fisher et al., "Effect of preoperative chemotherapy on local-regional disease in women with operable breast cancer: findings from National Surgical Adjuvant Breast and Bowel Project B-18.," Journal of clinical oncology, vol. 15, no. 7, pp. 2483-2493, 1997.

[5] I. R. Indran, G. Tufo, S. Pervaiz, and C. Brenner, "Recent advances in apoptosis, mitochondria and drug resistance in cancer cells," Biochimica et Biophysica Acta (BBA)Bioenergetics, vol. 1807, no. 6, pp. 735-745, 2011.

[6] E. A. Rakha et al., "Breast cancer prognostic classification in the molecular era: the role of histological grade," Breast Cancer Research, vol. 12, no. 4, p. 207, 2010.

[7] U. N. I. of Health, "National Cancer Institute, Cancer Statistics," Breast cancer incidence rates by race. Bethesda: US National Institutes of Health, 2007.

[8] C. B. Ambrosone et al., "Parity and breastfeeding among African-American women: differential effects on breast cancer risk by estrogen receptor status in the Women's Circle of Health Study," Cancer Causes \& Control, vol. 25, no. 2, pp. 259-265, 2014. 
[9] A. Stark, A. Kapke, D. Schultz, R. Brown, M. Linden, and U. Raju, "Advanced stages and poorly differentiated grade are associated with an increased risk of HER2/neu positive breast carcinoma only in White women: findings from a prospective cohort study of African-American and White-American women," Breast cancer research and treatment, vol. 107, no. 3, pp. 405-414, 2008.

[10]A. I. Phipps et al., "Reproductive history and risk of three breast cancer subtypes defined by three biomarkers," Cancer causes \& control, vol. 22, no. 3, pp. 399-405, 2011.

[11]P. Xing, J. Li, and F. Jin, "A case-control study of reproductive factors associated with subtypes of breast cancer in Northeast China," Medical Oncology, vol. 27, no. 3, pp. 926931, 2010.

[12]H.-L. Yang et al., "Growth inhibition and induction of apoptosis in MCF-7 breast cancer cells by Antrodia camphorata," Cancer letters, vol. 231, no. 2, pp. 215-227, 2006.

[13]K. Y. Yarso, I. W. Sudarsa, and I. B. T. Wibawa-Manuaba, "Clinical Initial Response of Neoadjuvant Chemotheraphy in Triple Negative, HER-2, and Luminal Types of Breast Cancer in Denpasar (A Preliminary Study)," Bali Medical Journal, vol. 1, no. 1, pp. 1216, 2012.

[14]R. Torrisi et al., "Tailored preoperative treatment of locally advanced triple negative (hormone receptor negative and HER2 negative) breast cancer with epirubicin, cisplatin, and infusional fluorouracil followed by weekly paclitaxel," Cancer chemotherapy and pharmacology, vol. 62, no. 4, pp. 667-672, 2008.

[15]G. Von Minckwitz et al., "Integrating bevacizumab, everolimus, and lapatinib into current neoadjuvant chemotherapy regimen for primary breast cancer. Safety results of the GeparQuinto trial," Annals of Oncology, vol. 22, no. 2, pp. 301-306, 2010.

[16]X.-H. Yang, T. L. Sladek, X. Liu, B. R. Butler, C. J. Froelich, and A. D. Thor, "Reconstitution of caspase 3 sensitizes MCF-7 breast cancer cells to doxorubicin-and etoposide-induced apoptosis," Cancer research, vol. 61, no. 1, pp. 348-354, 2001.

[17]N. O'Donovan et al., "Caspase 3 in breast cancer," Clinical Cancer Research, vol. 9, no. 2, pp. 738-742, 2003.

[18]S. Sharma, K. R. Hiran, K. Pavithran, and D. K. Vijaykumar, "A pilot study to assess the feasibility of evaluation of markers of response to chemotherapy at one day \& 21 days after first cycle of chemotherapy in carcinoma of breast: a prospective non-randomized observational study," World journal of surgical oncology, vol. 7, no. 1, p. 35, 2009. 